\title{
Gastric epithelioid haemangioendothelioma: a rare cause of upper gastrointestinal bleeding
}

\section{P Sanjay, S Raman, J Shannon, G T Williams, A Woodward}

Postgrad Med J 2005;81:e7 (http://www.postgradmedj.com/cgi/content/full/81/958/e7). doi: 10.1136/pgmj.2004.027367

Vascular tumours of the stomach are rare, representing $0.9 \%-3.3 \%$ of all gastric neoplasms. A 58 year old man was admitted as an emergency with a one day history of haematemesis and melaena. He underwent an emergency laparotomy for a tumour in the lesser curve of the stomach. The tumour showed the characteristic histological and immunohistochemical features of epithelioid haemangioendothelioma. Surgery in the form of wide excision seems to be the treatment of choice for this rare neoplasm. This case highlights the difficulty in diagnosing this rare tumour preoperatively and emphasises the need for long term follow up in view of its uncertain metastatic potential.

$\mathrm{V}$ ascular tumours of the stomach are rare, representing $0.9 \%-3.3 \%$ of all gastric malignancies. ${ }^{1}$ Epithelioid haemangioendothelioma (EHE) is one such tumour that can present as upper gastrointestinal bleeding and pose diagnostic difficulties. We report one such case in a 58 year old male patient who presented as an emergency with haematemesis and melaena. In this case report we highlight the rarity of this tumour, the difficulties in diagnosing it preoperatively, and the need for long term follow up in view of the lack of adequate literature regarding management and prognosis. A thorough literature search identified only two reported cases in English language journals $\mathrm{s}^{12}$ and this seems to be the first case to be recorded in the British medical literature.

\section{CASE REPORT}

A 58 year old white man presented as an emergency with a one day history of haematemesis and melaena with no medical history. Abdominal examination was unremarkable and he was haemodynamically unstable. An urgent gastroscopy after active resuscitation showed a $4 \mathrm{~mm}$ punched out ulcer along the lesser curvature of the distal body of the stomach. The bleeding was controlled with an injection of

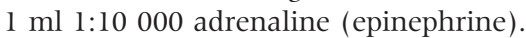

On the following morning the patient had a second episode of significant haematemesis requiring an urgent laparotomy. At operation, an indurated mass was felt in the distal body of the stomach with no other significant abnormality evident in the abdomen. A subtotal gastrectomy with a Roux-en-Y gastrojejunostomy was performed. The postoperative period was uneventful and the patient was discharged on the ninth postoperative day.

Macroscopic examination of the resected specimen showed a $5.0 \times 3.5 \times 1.5 \mathrm{~cm}$ polypoidal lesion on the lesser curve of the stomach (fig 1). Microscopic examination showed a cellular epithelioid neoplasm of the submucosa and muscularis propria lacking apparent mitotic activity $(0$ per 50 high power fields). Ki 67 staining confirmed a very low proliferation fraction. There was no evidence of epithelial dysplasia in the adjacent mucosa and none of the five lymph nodes retrieved contained metastatic tumour.

The initial histopathological diagnosis was thought to be gastrointestinal stromal tumour (GIST). However, further immunohistochemical studies showed that the tumour cells expressed several vascular markers such as CD31, CD34, and factor VIII related antigen, and CD31 staining highlighted the presence of intracytoplasmic lumina. There was also patchy expression of cytokeratin (fig 2). These findings pointed to

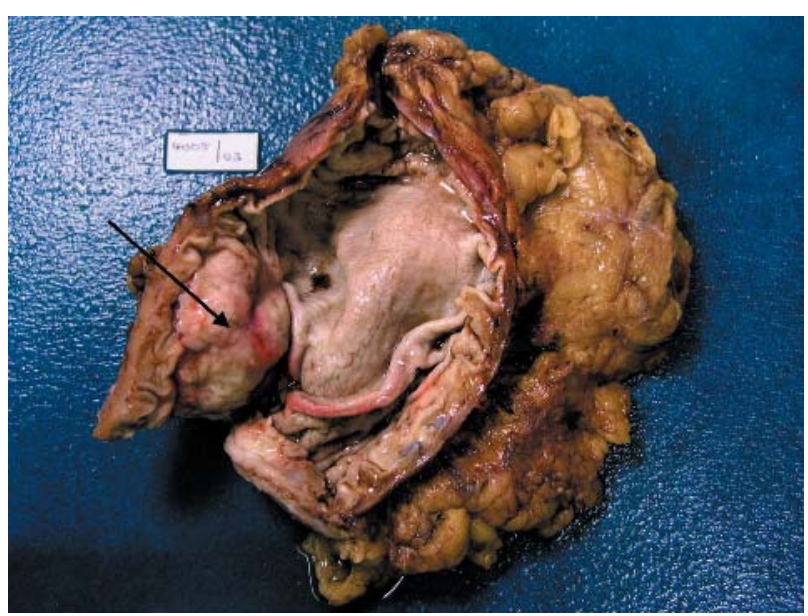

Figure 1 Stomach opened along the greater curve to show the tumour with overlying ulcerated mucosa.

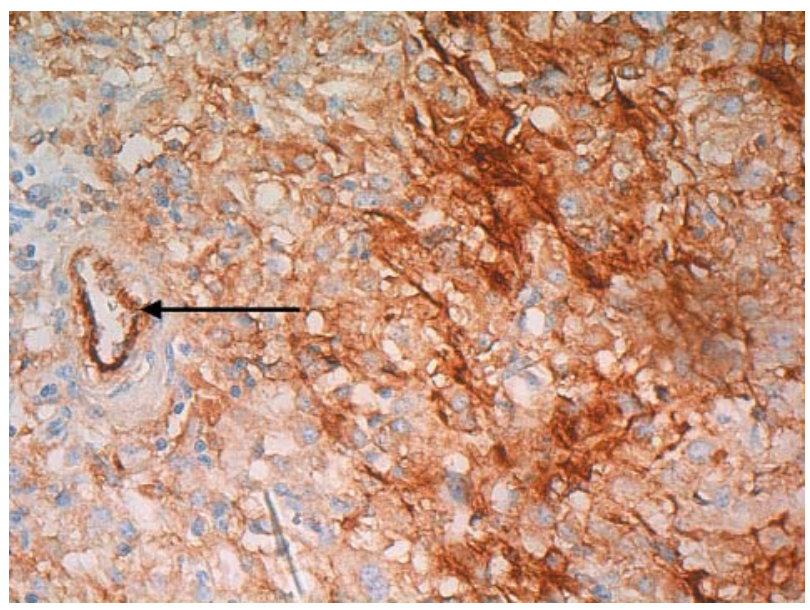

Figure 2 Immunostaining shows CD 34 positivity of many of the tumour cells (background vessels are suitably positive). 
the pathological diagnosis of an epithelioid haemangioendothelioma. Neural, muscle markers, and C-kit (CDI17, marker of GIST) were all negative.

A follow up gastroscopy and computed tomography of the abdomen at six months showed no evidence of local recurrence.

\section{DISCUSSION}

Epithelioid haemangioendothelioma is an uncommon but distinctive vascular tumour, which has been described in many sites such as the liver, ${ }^{3}$ lung, ${ }^{4}$ pleura, ${ }^{4}$ bone, ${ }^{5}$ omentum, ${ }^{6}$ and thyroid. ${ }^{7}$ This tumour is characterised by epithelioid or histiocytoid endothelial cell proliferation. The two previously reported gastric cases have occurred in middle aged adults. This tumour is usually of low grade malignancy but metastasis has been reported. ${ }^{8}$

The tumour can present as an abdominal mass or with clinical features of gastric outlet obstruction ${ }^{1}$ or upper gastrointestinal bleeding. ${ }^{2}$ Diagnosis by gastroscopy and biopsy is often inconclusive and difficult because of sampling difficulties related to the submucosal location of the tumour. ${ }^{1}$ Imaging techniques such as ultrasound or computed tomography may provide information about the location of the tumour, relation to neighbouring viscera, and the possibility of liver metastasis thus assisting in preoperative staging. ${ }^{9}$ Laparoscopy has also been reported to be helpful in identifying metastatic lesions in the liver, omentum, and peritoneum. $^{8}$

Histopathological examination supported by immunocytochemical studies are vital for the accurate diagnosis of this rare pathological entity. The combination of an "epithelioid" morphology of the tumour cells, the intracytoplasmic vacuoles, and the immunopositivity for markers of vascular differentiation including factor VIII related antigen, CD 34, and CD 31 help clinch the diagnosis. Other markers of muscle and neural differentiation are negative and a low proliferation fraction on Ki 67 staining helps to exclude angiosarcoma. Cases with a focal spindling of cells and areas of coagulative necrosis show greater potential for metastasis and recurrence. ${ }^{1}$ Similarly a high mitotic rate ( $>6$ mitosis/10 HPF) correlates with bad prognosis. ${ }^{9}$ In view of the fact that primary EHE is commoner in the liver, ${ }^{3}$ it is prudent to exclude a hepatic neoplasm before making a diagnosis of primary gastric EHE.

When a submucosal lesion is identified on endoscopy, wide excision of the tumour along with a $2 \mathrm{~cm}$ margin even without radical lymphadenectomy seems to be adequate. ${ }^{10}$ However, a mass lesion presenting as bleeding or obstruction may need to be treated as adenocarcinoma as preoperative diagnosis is difficult. Pleural EHE has been reported to respond to combination chemotherapy including carbaplatine and etoposide, ${ }^{4}$ but its value in gastric EHE is not known. The role of adjuvant chemotherapy and radiotherapy in malignant and recurrent disease remains to be established.

\section{CONCLUSION}

In summary this case report serves to highlight the existence of EHE of the stomach as a rare cause of upper gastrointestinal bleeding. It can cause diagnostic difficulties preoperatively as biopsies are inadequate because of the submucosal location of the tumour. Detailed immunohistochemical evaluation of the full specimen is essential to arrive

\section{Learning points}

- This case report serves to highlight the existence of EHE of the stomach as a rare cause of upper gastrointestinal bleeding.

- This tumour can cause diagnostic difficulties preoperatively as biopsies are inadequate because of its submucosal location.

- Immunohistochemistry plays a crucial part in the diagnosis of this pathological entity.

- Neoplasm is generally of low malignant potential with a good prognosis in the absence of histological markers of aggressiveness, however because of definite malignant potential long term follow up is recommended.

at a diagnosis. Based on the limited experience of surgical management obtained from review of the literature, ${ }^{12}$ wide excision seems to be adequate for cases with no histological markers of malignant potential (high mitotic figures, spindling of cells, necrosis).

The neoplasm is generally of low malignant potential with a good prognosis in the absence of histological markers of aggressiveness, however because of definite malignant potential long term follow up is recommended.

\section{Authors' affiliations}

P Sanjay, S Raman, A Woodward, Department of General Surgery, Royal Glamorgan Hospital, Llantrisant, UK

J Shannon, Department of Histopathology, Royal Glamorgan Hospital G T Williams, Department of Pathology, University Hospital Of Wales, Cardiff, UK

Correspondence to: Mr A Woodward, Department of General Surgery, Royal Glamorgan Hospital, Llantrisant, South Wales CF72 8XR, UK; drsanjay99@yahoo.co.uk

Submitted 29 July 2004

Accepted 3 November 2004

\section{REFERENCES}

1 Versaci A, Macri A. Epithelioid haemangioendothelioma of the stomach: clinical-pathological features, nosological setting, and surgical therapy. Report of a case. Surg Today 2002;32:274-7.

2 Lee KC, $\mathrm{Ng} \mathrm{WF}$, Chan JK. Epithelioid haemangioendothelioma presenting as a gastric polyp. Histopathology 1988;12:1065-8.

3 Lauffer JM, Zimmerman A. Epithelioid haemangioendothelioma of the liver. Cancer 1996:78:2318-27.

4 Pinet C, Magnan A. Aggressive form of pleural epithelioid haemangioendothelioma: complete response after chemotherapy. Eur Respir J 1999; 14:237-8.

5 Weiss SW, Kamal G. Epithelioid haemangioendothelioma and related lesions. Semin Diagn Pathol 1986;3:259-87.

6 Ratan S, Bhatnagar V. Epithelioid haemangioendothelioma of the greater omentum: report of a case. Surg Today 1999;29:919-21.

7 Siddiqui, Evans. Epithelioid haemangioendothelioma of the thyroid gland: a case report and review of literature. Histopathology 1998;32:473.

8 Seki K, Invi Y. A case of malignant haemangioendothelioma of the stomach. Endoscopy 1985; 17:78-80.

9 Mentzel T, Beham A. Epithelioid haemangioma of skin and soft tissues: clinicopathological and immunohistochemical study of 30 cases. Am J Surg Pathol 1997; 21:363-74.

10 Lehnert T. Surgical treatment of soft tissue tumours of the stomach. Eur J Oncol $1990 ; 16: 352-9$. 